\title{
ZUR ETYMOLOGIE VON LAT. LAXUS 'LOCKER, WEIT’
}

\section{Stefan Höfler}

Harvard University, Department of Linguistics

Boylston Hall, $3^{\text {rd }}$ floor Cambridge, MA 02138

United States of America; hoefler@fas.harvard.edu

The aim of this contribution is to demonstrate that the commonly accepted etymological link between Lat. laxus 'wide, spacious, loose' and Lat. languere 'be faint, weak' is not only formally difficult, but also semantically unjustified. Discussing the earliest attestations and derivatives of laxus against the backdrop of general morphosemantic considerations, I propose deriving this adjective and the enigmatic Lat. locus $\mathrm{m}$. 'place' from the root ${ }^{*} \sqrt{s l e k}$, whose possible semantics and continuants in other IE languages are dealt with in the remainder of the paper.

Keywords: Latin Etymology, laxus, langueō, locus, Possessive Adjectives, Schwa secundum.

Anhand der gängigen Hand- und Wörterbücher, die sich - wenn auch nur entfernt - mit lateinischer Sprachwissenschaft und Etymologie befassen, könnte man durchaus den Eindruck gewinnen, die sprachhistorische Verwandtschaft und etymologische Zusammengehörigkeit des Adjektivs lat. laxus 'locker, weit' (Cato+) mit dem Verbum langueō 'bin matt, schlaff, unlustig; welke' (Lucil.+) sei in Stein gemeißelt: $c f$. Walde/Hofmann s.v. langueō (laxus als ${ }^{*}(s)$ log-sos); Ernout/Meillet s.v. laxus („forme désidérative à élargissement -s-"); IEW, 959-960; TLL s.v. laxus (,fortasse eiusdem radicis ac langueo"); OLD s.v. laxus; Schrijver 1991, $136 \mathrm{f}$ u. $165\left({ }^{*}(s) l h_{2 g}\right.$-so-); de Vaan 2008 s.v. laxus ( ${ }^{*}$ slg-somit Verweis auf "Schrijver's rule $\left.{ }^{\star} R D C>{ }^{\star} R a D C^{“ 1}\right)$ und s.v. langueō; Matasović 2009 s.v. ${ }^{*}$ laggo- (mit Verweis auf ein lat. „languo" [sic]).

Bei genauerem Hinsehen erweist sich diese Verbindung jedoch weit weniger attraktiv als sie anfangs vielleicht erschienen sein mochte, da sich die Bedeutungen und Verwendungssphären von laxus einerseits und languēre andererseits nur dann überlappen, wenn eine verdächtig übereinstimmende Glossierung ('schlaff' bzw. 'schlaff sein' vel sim.) gewählt wird; in den primären Textzeugnissen kann eine solche Bedeutungsgleichheit allerdings nicht ausgemacht werden - languère bedeutet zwar sehr wohl 'schlaff, matt sein (von Personen)', laxus dagegen aber vielmehr 'locker, weit, geräumig (von Dingen)'.

Cato liefert uns den ersten Beleg für das Adjektiv in seiner De agri cultura, wo es in 20,2 heißt: eos (sc. modiolos) circumplumbato, caueto ne laxi sient „[U]mgieße sie [sc. die Naben für die Olivenquetschsteine] mit Blei. Gib acht, dass sie nicht locker sind“2, wobei schon hier ersichtlich wird, dass weniger von einem 'erschlafften' als von einem 'weiten, geräumigen' Zustand der assoziierten Substantive (hier: der modioli 'Naben') die Rede ist, wie dies auch für weitere ausgewählte, allesamt relativ frühe Belege der Fall ist: Uolusi annales ... laxas scombris saepe dabunt tunicas (Catull. 95, 7-8): „Die Annalen des Volusius werden den Makrelen als lockere Hülle dienen“ (nicht: „erschlaffte Hülle“); commodissi-

${ }^{1} \mathrm{Zu}$ dieser Regel $c f$. Schrijver 1991, 485. Gerade für laxus setzt dieser Forscher jedoch eine laryngalhaltige Vorform an (siehe im Text zuvor).

2 Übersetzung nach Hartmut Froesch, Stuttgart: Reclam, 2009. Wenn nicht anders angegeben, stammen die Übersetzungen in weiterer Folge vom Verfasser.

(C) St. Petersburg State University, 2017 
mum esse quam laxissimas habenas habere amicitiae (Cic. Lael. 45): „...dass es am besten sei, in Bezug auf eine Freundschaft möglichst weite Zügel zu haben“ (nicht: „möglichst schlaffe Zügel“"3); uillam ponat, ubi pastiones sint laxae (Varro Rust. 1, 12, 1): „Das Gehöft baue er dort, wo es weitläufige Weideflächen gibt“.

Dagegen würde eine Herleitung aus der Sippe rund um languēre (cf. als Erstbeleg etwa Acc. trag. 637-638: iam iam stupido Thessala somno / pectora languentque senentque „Und schon sind die thessalischen Gemüter durch Schlummer schlaff und matt"), das im $L I V^{2}$ auf p. 565 zusammen mit gr. $\lambda \alpha \gamma \gamma \omega \dot{\omega} v$ 'Feigling' (als Partizip eines Nasalinfixpräsens) zu einer Wurzel ${ }^{*} \sqrt{ }$ sleg $/ \hat{g}$ 'erschlaffen' gestellt wird (vgl. auch an. slakr 'schlaff' etc. < ${ }^{{ }^{*}}$ log $/ \hat{g}$-o-), und zwar als Verbaladjektiv ${ }^{4}{ }^{*} l_{\partial} g / \hat{g}$-so-für laxus ja eine Grundbedeutung 'erschlafft' voraussetzen, von der jedoch kaum ein Weg zu tatsächlich belegtem 'locker, weit, geräumig' führt, wie ja aus den zuvor genannten laxus-Belegen ersichtlich ist. Es ist viel plausibler, die umgekehrte Entwicklungsrichtung anzunehmen, nämlich dass sich eine Grundbedeutung 'weit, geräumig, Platz habend' (cf. OLD, 1012 s.v. laxus Bed. 1 „Spacious, wide, ample, roomy“, Bed. 2 „Not closely packed, loose in arrangement or structure“) in bestimmten Kontexten zu 'locker, schlaff' (cf. OLD, 1012 s.v. laxus Bed. 6 „(of parts of the body) Relaxed, loose") entwickelt hat; die Reihung der Bedeutungen im OLD gibt ja Aufschluss darüber, dass das in einer Untermenge der anfangs genannten Referenzwerke als Grundbedeutung suggerierte 'schlaff' tatsächlich nur eine marginal belegte semantische Nuance darstellt.

Für eine Grundbedeutung 'Platz habend, geräumig' sprechen zudem ganz entschieden die Ableitungen laxitās, -ātis f. 'Weite, Geräumigkeit; freier Raum; Bewegungsfreiheit; Gelassenheit' (Cic.+), laxō, -äre 'mache weit, breite aus; lockere auf; lasse frei; entspanne, spanne aus etc.' (Cic.+), laxāmentum, -ī n. 'Schonung, Spielraum, Erweiterung; der (weite) Raum' (Cato+), das Adverb laxē 'lose, uneingeschränkt, weit, geräumig' (laxē habitäre 'in einem geräumigen Haus wohnen' Cic.), sowie die Glossierung von Nonius p. 340, 6 laxum: liberum et remissum ... non angustum, sed spatii maioris ,laxum [heißt] frei und locker (...) nicht eng, sondern mit viel Platz dazwischen“. Gewiss kann als weiteres Argument gegen eine Verwandtschaft von languère und laxus auch der Umstand gewertet werden, dass das Verbum prototypisch Lebewesen zum Subjekt hat ${ }^{5}$, umgekehrt laxus jedoch just nie (!) auf Menschen (sondern höchstens auf Körperteile ${ }^{6}$ ) bezogen

${ }^{3}$ Dass laxissimas habenas hier nicht „möglichst schlaffe, lose Zügel“ bedeutet, wird durch den darauffolgenden Zusatz klar, wo es nämlich heißt quas uel adducas, cum uelis, uel remittas, also „die du nach Belieben entweder straffziehst oder schießen lässt" (in diesem Sinne auch bereits Nauck 1897, 48).

${ }^{4}$ Es wäre eine Ersatzbildung auf -so- (wie fìxus neben fïctus, luxus neben *luctus [cf. luctāri], ferner läpsus, etc.) statt eines erwarteten ${ }^{*}(s) l_{\partial} g$-to- 'erschlafft' anzusetzen.

5 OLD, 999 s.v. langueō Bed. „1 (of persons, parts of the body, etc.) To be physically sluggish or faint, be enfeebled; (also of physical faculties, etc.). $\mathbf{b}$ (spec.) to be unwell, be sick. $\mathbf{c}$ to be languid or torpid (from sleep). (...) 3 To be disinclined for action, be idle or inert (...)" mit Beispielen.

${ }^{6}$ Nach OLD, 1012 s.v. laxus Bed. 6 vor allem von Persius, z.B. 1, 98: quidnam igitur tenerum et laxa ceruice legendum? „Was nun gilt als geschmeidig, als lesbar lässigen Nackens?“, oder 3, 58-59: stertis adhuc laxumque caput conpage soluta / oscitat hesternum dissutis undique malis „Du aber schnarchst. Dein schlottriger Schädel, noch ganz aus den Fugen, / Gähnt vom gestrigen Rausch, und die Kinnlad' geht aus der Naht schier!“ (Übersetzung nach Otto Seel, München 1950). Wenn sich laxus dann doch auf Menschen bezieht, ist dies stets als Anspielung auf zu häufigen oder zu intensiven Geschlechtsverkehr zu verstehen (cf. TLL s.v. laxus Bed. $1 \mathrm{~b}$ „de hominibus c. respectu earum partium, quae coitu frequenti dilatatae sunt" mit Mart. 11, 21, 1: Lydia tam laxa est equitis quam culus aeni „Lydia ist so ausgeweitet wie der Hintern einer bronzenen Reiterstatue" (Übersetzung nach Paul Barié und Winfried Schindler, Berlin: Akademie Verlag, 2013³) und 
wird, ${ }^{7}$ was eine etymologische Zusammengehörigkeit zwar nicht widerlegt, jedoch zumindest ernsthaft infrage stellt.

Haben wir uns erst einmal von der irrigen Assoziation mit languēre befreit, gilt es nun, für laxus nach einer plausibleren Herleitung Ausschau zu halten. Infrage käme, und zwar sowohl auf lautlicher wie auch auf semantischer Ebene, eine etymologische Anbindung an lat. locus, -i m. 'Platz, Ort, Stelle', das innerhalb des Lateinischen sonst isoliert dasteht und in der Vergangenheit oftmals fälschlicherweise als ${ }^{*}$ stloko- rekonstruiert worden ist. Ja, die von (inter alios) Walde/Hofmann I, 817f und IEW, 1019 sowie NIL, 662f m. Anm. 2 (m. Lit.) propagierte, auf einer Missinterpretation der Nebenform stlocus (Quint., Paul. Fest. 312) basierende Zusammenstellung zur Wurzel ${ }^{\star} \sqrt{ }$ stel 'hinstellen, bereit machen' ( $L I V^{2}$, 594) als ${ }^{*}$ stl-o-ko-vel sim. ${ }^{8}$ wird von de Vaan 2008,347 ganz richtig und mit gutem Grund abgelehnt. Kann anlautendes stl- doch auch mit anaptyktischem Dental auf eine Lautfolge ${ }^{*}$ sl- zurückgehen ( $c f$. lìs neben stlīs und sLIS [CIL 12 1 .583, par. 8, 123/122 v.d.Z.] f. 'Streit; Rechtsstreit' ${ }^{\text {') }}$, was für lat. locus eine morphologisch durchsichtigere Vorform ${ }^{*}$ slok-o- (so auch de Vaan loc. cit.) wahrscheinlich macht.

Lat. laxus könnte dann eine Possessivableitung ${ }^{\star}$ slak-s-o- $<{ }^{*}$ slo $k$-s-ó- (lautlich mit Schrijver 1991, 485) oder eher ${ }^{\star} s l_{2} k$-s-ó- (mit Schwa secundum) zu einem neutralen $s$-Stamm ${ }^{\star}$ slék-os derselben Wurzel ${ }^{\star} \sqrt{ }$ slek darstellen, der in seiner Semantik wohl nur gering von seinem thematischen Gegenstück ${ }^{*}$ slok-o- 'Platz, Ort, Stelle'10 abgewichen sein dürfte ( $c f$. pondus, -eris n. 'Gewicht' neben thematischem, gleichbedeutendem *pondo-in Adv. pondō 'an Gewicht'; oder umbr. meřs n. 'ius' (lat. modes-tus) neben lat. modus, -ī m. 'Maß').

Man vergleiche zur Bildung von denominalen Possessivableitungen mittels des Suffixes *ó- auf Basis neutraler s-Stämme die Beispiele *léuk-os n. 'Licht' (aav., jav. raocah- n. 'Licht, Tag') $\rightarrow{ }^{\star}$ luk-s-ó- 'Licht habend' (ved. rukșá- 'glänzend' $R V$ 6.3.7), oder

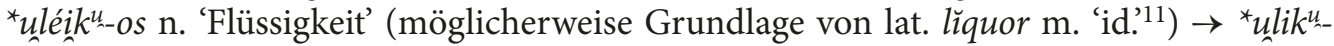
$s$-ó- 'Flüssigkeit habend' (substantiviert als kymr. gwlych 'Flüssigkeit'; lat. Lixus m. 'ein Flussname' und lat. lixa f. 'Wasser, Lauge'). ${ }^{12}$ Noch ein Beispiel aus dem Lateinischen wäre das Adjektiv alsus 'kühlend, erfrischend' (Cic.), das meiner Ansicht nach ein ${ }^{\star} h_{2}(e) l g^{h}-s$-ó'Kälte habend' zum $s$-Stamm ${ }^{\star} h_{2} e ́ l g{ }^{h}$-os n. 'Kälte' ( $c f$. lat. algor m. 'id.' Plaut.+; nisl. elgur m. 'Schneegestöber; halbgeschmolzener Schnee') fortführt. ${ }^{13}$ Obwohl wir vorerst keine Evidenz für einen $s$-Stamm ${ }^{*}$ slék-os 'Raum' vorweisen können, ist zumindest die formale Seite unserer Herleitung durch diese Beispiele gestützt.

Passenderweise liefern, was die semantische bzw. morphosemantische Facette unserer Etymologie betrifft, nicht nur moderne Sprachen wie das Englische (room $\rightarrow$

weiteren Beispielen), wo eine Deutung 'schlaff' < 'erschlafft' nicht möglich ist, sondern vielmehr auf (ugs.) 'ausgeleiert' < 'weit' angespielt wird. Cf. dazu auch Obermayer 1998, $184^{179}$.

7 Dies dann ein zusätzlicher Grund, die von Érnout/Meillet befürwortete Herleitung aus einem desiderativen Verbalstamm (siehe ganz zu Beginn) abzulehnen.

${ }^{8}$ Diese Form wäre ja ein morphologisches Ungetüm.

9 Cf. Schindler apud Joseph 1986, $122 \mathrm{f}$ mit Belegen; Gusmani 2002 (mit Anknüpfung an germ. ${ }^{*}$ sleit'zerreißen, spalten'); de Vaan 2008, 345f; Weiss 2009, 177 m. Fn. 4.

${ }_{10}$ Zur Bedeutung 'Platz, zur Verfügung stehender Raum', die hier ja vorausgesetzt wird, $c f$. OLD s.v. locus Bed. 12 „Extent of ground, surface, etc.; (usu.) extent sufficient for some purpose, room. b [loc] um dare, to give place, make way ... c continuous extension, space." mit Beispielen aus (u.a.) Plautus, Terenz, Lukrez.

${ }_{11}$ Wohl eher innerlat. Neubildung zu liquēre 'flüssig sein'.

${ }^{12}$ Vgl. zu diesen Bildungen Höfler 2015.

13 Ähnlich bereits Brugmann 1906, 541. 
roomy) oder das Deutsche (Raum $\rightarrow$ geräumig) Parallelbeispiele für den implizierten Prozess, auch innerhalb des Lateinischen selbst ist mit spatium n. 'Raum' $\rightarrow$ spatiōsus 'geräumig, weit' ein ganz ähnlich gelagerter Fall belegt, wobei das abgeleitete Adjektiv u.a. in genau denselben Kontexten wie unser laxus verwendet werden kann. Quintilian sagt Inst. 11,18 loca ... quam maxime spatiosa "möglichst weite Gebiete" und entspricht in diesem Gebrauch der von Columella verwendeten Junktur in 7, 12, 10 si tam laxa rura sunt „wenn die Ländereien so weitläufig sind“. ${ }^{14}$

Rein theoretisch käme als Grundbedeutung für die Verbalwurzel, von der ${ }^{\star}$ slók-o- m. und ${ }^{*}$ slék-os n. offenkundig abgeleitet sind, eine Semantik 'Raum haben' infrage, wie sie etwa auch für die Wurzel ${ }^{*} \sqrt{t e l p}\left(I E W, 1062 ; L I V^{2}, 623\right.$ als 'Raum schaffen') angesetzt wird; cf. lit. telpù 'finde Raum, habe Platz', air. nítella 'hat nicht Platz'15, lit. talpà f. 'ausreichender Raum, Rauminhalt', ved. tálpa- m. 'Lager, Bett' $(A V+)$. Sonstige, im Litauischen zu dieser Sippe gehörige Bildungen umfassen u.a. die Begriffe talpùs 'geräumig, umfangreich' (dies dann semantisch mit laxus vergleichbar) und talpyklà f. 'Behälter, Gefäß'16. Tatsächlich liefert letzteres eine semantische Rechtfertigung für die Annahme, dass sich eine Nominalform zu einer Wurzel mit der Semantik 'Raum haben' als Begriff für eine Gefäßbezeichnung etablieren konnte. Und vor diesem Hintergrund lässt sich sodann

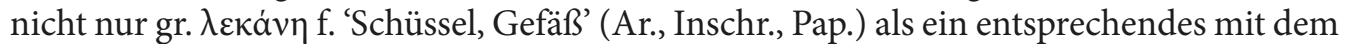
bei Gerätbezeichnungen nicht unüblichen Suffix - $\alpha \dot{v} \eta^{17}$ abgeleitetes Substantiv zur Wurzel ${ }^{*} \sqrt{s l e k}$ deuten, sondern zudem der dem Adjektiv lat. laxus vermeintlich zugrundeliegende $s$-Stamm ${ }^{*}$ slék-os n. im Neutrum gr. $\lambda \dot{\varepsilon} \kappa o \varsigma$ n. 'Schüssel, Gefäß' (Hippon. u.a.) erkennen, wodurch die Plausibilität des Rekonstrukts ${ }^{*} s l_{\partial} k$-s-ó- entscheidend an Boden gewinnt. Vermutlich lief bei diesem $s$-Stamm die semantische Entwicklung von 'Raum(inhalt), Fassungsvermögen' über 'konkretes Fassungsvermögen' zu 'Gefäß, Schüssel'.

Wir können also auf allen Ebenen, sowohl philologisch wie auch formal und morphosemantisch, durchaus zuversichtlich sein, was die hier neu vorgelegte Etymologie von lat. laxus betrifft. X-beliebig ließe sich zwar die Liste der semantischen Parallelen für die involvierten Prozesse fortführen, doch sei hier lediglich noch darauf hingewiesen, dass die Grundbedeutung der Wurzel ${ }^{*} \sqrt{s l e k}$ durchaus auch eine andere gewesen sein konnte; ein Vergleich mit dem $s$-Stamm ved. vyácas- n. 'Umfang, Ausdehnung, Fassungsvermögen'18 $(R V+)$ bzw. '(umfänglicher, weiter) Raum'19 (qua (morpho-) semantische Entsprechung vom $s$-Stamm ${ }^{*}$ slék-os) und der Possessivableitung vyácasvant- 'ausgedehnt' (qua (morpho-)semantische Entsprechung von ${ }^{*}{ } l_{\partial} k-s-o ́->$ lat. laxus),

14 Etwa mit tempus (Ov. Am. 1, 8, 81: nunquam dederis spatiosum tempus in iram, und Plin. Ep. 4,9,14: tempus sibi et quidem laxius uindicauit); mit domus (Plin. Ep. 7, 12, 7: spatiosa et capax domus, und Vell. 2,81,3: quo laxior fieret ipsius [domus]); mit orbis (Ov. Am. 3, 2,6 9: metam spatioso circuit orbe, und Sen. Nat. 1,1,2: fieri primum angustissimum orbem, deinde laxiorem), mit uolumina (Manil. 1,335: laxa uolumina [serpentis], und Luc. 3, 505: nigri spatiosa uolumina fumi); etc. Interessant sind auch folgende Phrasen und Belege: spatiosior laxitas (Plin. Nat. 2,218); laxo spatio (Liv. 10, 5, 6); riguus locus spatia laxiora desiderat „Ein feuchter Ort verlangt nach größeren Abständen [zwischen den Setzlingen]“ (Col. 4, 30, 5); si ad cogitandum uoltis sumere tempus sumite laxsi spatii (BGU 2,611,3,14).

15 Schumacher 2004, 506-507.

16 Cf. Smoczyński 2007, 678. Zum Suffix (aus *-tleh ${ }_{2}$ ) cf. Leskien 1891, 500; Skardžius 1943/1996, 195-196.

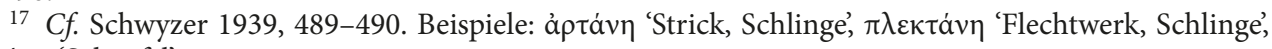
бкат⿱亠乂⿰丿 ‘'Schaufel', etc.

18 Cf. EWAia II, 590.

19 Cf. PW s.v. vyácas-. 
die zu einer Wurzel $V_{\text {uiek }}$ ' 'umwinden, umspannen' (LIV 2 , 696; VIA, 399; cf. ved. vivyāca 'umfasst', lat. uincìre 'schnüren, binden') gehören, zeigt, dass durchaus auch eine andere Wurzelsemantik ('umfassen', 'messen', vel sim.) infrage käme, doch solche Spekulationen würden momentan (bzw. solange keine verbale Evidenz für die hier postulierte Wurzel gefunden ist) keinen Gewinn bringen und nur nach York bzw. zum Ausgangspunkt unserer Untersuchung zurückführen. York war ja bekanntlich die Heimat des einem Kinderreim entsprungenem "grand old Duke“, der seine zehntausend Mann starken Truppen auf einen Hügel ziehen ließ, nur um sie später von selbigem wieder an den Ursprungsort zurückzuführen. Ziel des vorliegenden Beitrags war es aber lediglich aufzuzeigen, dass die herkömmliche etymologische Anbindung des Adjektivs lat. laxus an das Verbum langueō nicht nur formal schwierig, sondern auch semantisch kaum attraktiv ist, was mir durch das Aufzeigen einer plausiblen und in sich stimmigen Alternative im Urteil der aufmerksamen Leserschaft — so hoffe ich zumindest — gelungen sein mag.

\section{Literaturhinweise}

Brugmann K. Vergleichende Laut-, Stammbildungs- und Flexionslehre nebst Lehre vom Gebrauch der Wortformen der indogermanischen Sprachen. 2. Bearb., 2. Band, Erster Teil. Strassburg, Trübner, 1906.

Ernout/Meillet: A. Ernout \& A. Meillet. Dictionnaire Étymologique de la Langue Latine. Histoire des Mots. Paris, Klincksieck, ${ }^{4} 1985$.

EWAia: M.Mayrhofer. Etymologisches Wörterbuch des Altindoarischen. 3 Bde. Heidelberg, Winter, 1992-2001.

Gusmani R. Althochdeutsch (hari)sliz, lateinisch (st)lis. Historische Sprachforschung 2002, 115, 111-116.

Höfler S. Denominale Sekundärderivation im Indogermanischen: Eine Ochsentour. Münchener Studien zur Sprachwissenschaft 2015, 69/2, 219-243.

IEW: J. Pokorny. Indogermanisches Etymologisches Wörterbuch. I. Band. Bern, Francke, ${ }^{2} 1989$.

Joseph L. A survival from the Italo-Celtic legal vocabulary. Ériu 1986, 37, 119-125.

Leskien A. Die Bildung der Nomina im Litauischen. Leipzig, Hirzel, 1891.

LIV $V^{2}$ : H. Rix. Lexikon der indogermanischen Verben. Die Wurzeln und ihre Primärstammbildungen. Wiesbaden, Reichert, ${ }^{2} 2001$.

Matasović R. Etymological Dictionary of Proto-Celtic. Leiden, Brill, 2009.

Nauck C.W. M. Tulli Ciceronis Laelius De Amicitia. Erklärt von C. W. Nauck. 10. Aufl. Besorgt von Th. Schiche. Berlin, Weidmannsche Buchhandlung, 1897.

NIL: Nomina im Indogermanischen Lexikon. Hg. von D. S. Wodtko, B. Irslinger, C. Schneider. Heidelberg, Winter, 2008.

Obermayer H.P. Martial und der Diskurs über die männliche "Homosexualität" in der Literatur der frühen Kaiserzeit. Tübingen, Narr, 1998.

PW: Sanskrit-Wörterbuch. Hg. von der Kaiserlichen Akademie der Wissenschaften, bearb. von O. Böhtlingk und R. Roth. 7 Bde. St. Petersburg, Kaiserliche Akademie der Wissenschaften, 1855-1875.

Schrijver P. The Reflexes of the Proto-Indo-European Laryngeals in Latin. Amsterdam, Rodopi, 1991.

Schumacher S. Die keltischen Primärverben. Ein vergleichendes, etymologisches und morphologisches Lexikon. Innsbruck, IBS, 2004.

Schwyzer E. Griechische Grammatik. Auf der Grundlage von K. Brugmanns Griechischer Grammatik. 1. Allgemeiner Teil. Lautlehre, Wortbildung, Flexion. München, Beck, 1939.

Skardžius P. Lietuviu kalbos žodžiu daryba. Rinktiniai raštai 1. Vilnius, Mokslo ir enciklopedijų leidykla, 1943/1996.

Smoczyński W. Lietuvių kalbos etimologinis žodynas. Vilnius, 2007.

TLL: Thesaurus Linguae Latinae (TLL) Online (2009), Berlin, Boston: De Gruyter, http://www.degruyter. $\mathrm{com} / \mathrm{view} / \mathrm{db} / \mathrm{tll}(31.08 .2017)$.

de Vaan M. Etymological Dictionary of Latin and the other Italic Languages. Leiden, Brill, 2008.

VIA: C. H. Werba. Verba IndoArica. Die primären und sekundären Wurzeln der Sanskrit-Sprache. Pars I: Radices Primariae. Wien, Verlag der österreichischen Akademie der Wissenschaften, 1997. 
Walde/Hofmann: A. Walde \& J.B.Hofmann. Lateinisches Etymologisches Wörterbuch. 2 Bde. 3., neubearb. Aufl. Heidelberg, Winter, 1938-54.

Weiss M. Outline of the Historical and Comparative Grammar of Latin. Ann Arbor, New York, Beech Stave, 2009.

For citation: Höfler S. Zur Etymologie von lat. laxus 'locker, weit'. Philologia Classica 2017, 12(2), 154159. https://doi.org/10.21638/11701/spbu20.2017.205

\section{К ЭТИМОЛОГИИ ЛАТИНСКОГО LAXUS 'ШИРОКИЙ, СВОБОДНЫЙ’}

\section{Стефан Хёблер}

Цель статьи - показать, что общепринятая этимология лат. laxus 'широкий, просторный, свободный', основанная на связи с лат. languère 'быть слабым, вялым', не только сталкивается с формальными трудностями, но и слабо обоснована семантически. Основываясь на ранних свидетельствах и производных laxus, а также на общих морфосемантических соображениях, автор статьи предлагает связывать это прилагательное с трудно этимологизируемым лат. locus m. 'место', возводя оба слова к корню * $\sqrt{s l e k}$, предполагаемые семантика и производные которого в других индоевропейских языках рассматриваются в статье.

Ключевые слова: латинская этимология, laxus, langueō, locus, притяжательные прилагательные, schwa secundum. 